\title{
Pyridoxine dependent epilepsy: a suggestive electroclinical pattern
}

\author{
R Nabbout, C Soufflet, P Plouin, O Dulac
}

\begin{abstract}
Aims-To determine if there is an electroencephalographic pattern suggestive of pyridoxine dependent epilepsy that could be used to improve the chances of early diagnosis.

Methods-A retrospective study was made of all the clinical records and electroencephalograms of neonates identified with pyridoxine dependent seizures between 1983 and 1994, at this hospital. Neonates whose seizures began after more than 28 days of life were excluded; in all, five patients from four families were studied. Follow up ranged from 2 to 10 years.

Results-A history of miscarriage and neonatal death during an epileptic seizure had occurred in the siblings of two families. One mother reported rhythmic movements of her child during the last month of pregnancy. At birth, all babies were hypotonic; four had decreased visual alertness. All babies were agitated, irritable, jittery, hyperalert, and exhibited sleeplessness and a startle reaction to touch and sound. Age of onset of seizures varied from 30 minutes to 3 days. Seizures of various types were recorded in all cases on EEG tracings, including spasms, myoclonic seizures, partial clonic, and secondary generalised seizures. Burstsuppression patterns occurred in three cases, and a combination of continuous and discontinuous patterns in two others. Bilateral high voltage delta slow wave activity was observed in four patients. Psychomotor delay was severe in three patients, moderate in one, and mild in one.
\end{abstract}

Conclusions-There is an identifiable EEG pattern that is highly suggestive of pyridoxine dependent epilepsy. Pyridox-

Department of Neuropaediatrics R Nabbout

O Dulac

Department of

Neurophysiology

C Soufflet

P Plouin

INSERM U 29

Université René

Descartes

Hospital Saint Vincent

de Paul

82 avenue Denfert

Rochereau

75674 Paris cx 14

France

O Dulac

Correspondence to: Dr O Dulac. ine dependent epilepsy is probably underdiagnosed.

(Arch Dis Child Fetal Neonatal Ed 1999;81:F125-F129)

Keywords: pyridoxine; convulsions; genetics; epilepsy

Pyridoxine dependency is a rare autosomal recessive disorder causing severe neonatal epilepsy. Since it was first described by Hunt et al in $1954,{ }^{1}$ fewer than 100 cases have been reported. Seizures usually occur during the first few days of life, ${ }^{2-5}$ but these may occur either in utero ${ }^{6}$ or subsequently in infancy or early childhood. ${ }^{6-9}$ Although the underlying defect remains unknown, pyridoxine dependent seizures seem to be due to deficiency of brain gamma aminobutyric acid (GABA), a major inhibitory neurotransmitter of the central nervous system. Pyridoxal phosphate (PLP), the active metabolite of pyridoxine, is the coenzyme for glutamate decarboxylase (GAD), the rate limiting enzyme for the production of GABA from glutamate. A glutamate excess may also be neurotoxic in this condition. ${ }^{10}$

Pyridoxine dependency is usually suspected in cases of recurrent and intractable neonatal seizures. As no laboratory test is available to confirm the diagnosis, it is always delayed and made after several weeks, except, of course, in familial cases. Pyridoxine administration is the only way of stopping seizures and thus of confirming the diagnosis. Most infants exhibit subsequent mental retardation. Nevertheless, early treatment might decrease the severity of intellectual deficit.

This retrospective study aimed to identify a characteristic electroclinical pattern, to contribute to early diagnosis and treatment, and the prevention of subsequent mental retardation. 
Table 1 Clinical details of patients studied

\begin{tabular}{lllllllll}
\hline $\begin{array}{l}\text { Case } \\
\text { Nos }\end{array}$ & Consanguinity & $\begin{array}{l}\text { Term birth by week } \\
\text { of gestation }\end{array}$ & $\begin{array}{l}\text { Fetal } \\
\text { distress }\end{array}$ & $\begin{array}{l}\text { Intestinal ileus } \\
\text { at birth }\end{array}$ & $\begin{array}{l}\text { Onset of } \\
\text { convulsions }\end{array}$ & $\begin{array}{l}\text { Status } \\
\text { epilepticus }\end{array}$ & $\begin{array}{l}\text { Onset of } \\
\text { treatment }\end{array}$ & $\begin{array}{l}\text { Psychomotor } \\
\text { delay }\end{array}$ \\
\hline 1 & - & 35 & + & + & 30 minutes & - & 58 days & Severe \\
2 & + & 37 & + & - & 6 hours & + & 18 days & Moderate \\
3 & + & 38 & - & + & 3 days & + & 10 days & Severe \\
$4^{\star}$ & + & 38 & - & - & 24 hours & + & 30 days & Severe \\
$5^{\star}$ & + & 37.5 & - & - & 4 days & - & 4 days & Mild \\
\hline
\end{tabular}

These two patients are brothers.

\section{Methods}

The clinical records and electroencephalograms (EEG) were reviewed of patients identified as having had pyridoxine dependent seizures in the neonatal period, and referred to Saint Vincent de Paul Hospital between 1983 and 1994. Patients whose seizure onset began after 28 days of life were excluded. Five patients belonging to four families were identified. Three were consanguineous, and a history of miscarriage and neonatal death during status epilepticus in siblings was present in two families (fig 1). In one case the mother described rhythmic movements of her child during the last month of pregnancy.

\section{Results}

All births except one were born at term. Two neonates had fetal distress with meconium stained fluid, but did not require admission to intensive care. On examination at birth they were hypotonic, with decreased visual alertness in four. No dysmorphic features were noted. Birthweight, height, and head circumference were within the normal range.

All babies exhibited agitation, irritability, jitteriness, hyperalertness and sleeplessness, as well as the startle reaction to touch and sound.

Two patients had ileus with distended abdomen followed by spontaneous remission after one week of nasogastric tube feeding. In the preterm child ( 35 weeks of gestation), an intestinal dilatation had been diagnosed by systematic in utero ultrasonography but no surgical condition had been diagnosed.

The age of onset of seizures ranged from 30 minutes to 3 days (table 1 ). The babies exhibited a great variety of intermixed seizures consisting of spasms, myoclonus, partial clonic and secondary generalised seizures. The two latter types evolved into status epilepticus within the first few days of life in three neonates (table 1).

EEG FINDINGS

All patients had EEG tracings before pyridoxine treatment, but the interpretation was often difficult because of movement artefacts due to hyperexcitability and unremitting agitation. Interictal EEG disclosed no normal background activity, except for short runs of regular 4 to $5 \mathrm{~Hz} \theta$ activity. No sleep organisation existed. Three neonates exhibited discontinuous EEG with suppression burst-like pattern (fig 2), the suppression periods lasting up to 14 seconds in one patient who was receiving high doses of clobazam (fig 3). Two neonates had a more polymorphous tracing, with a combination of continuous and discontinuous patterns. Spikes and polyspike foci were recorded in

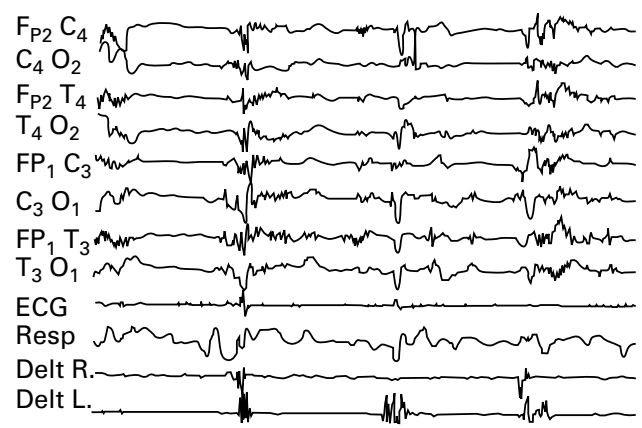

Figure 2 Discontinuous suppression burst-like pattern in the EEG tracings of three neonates.

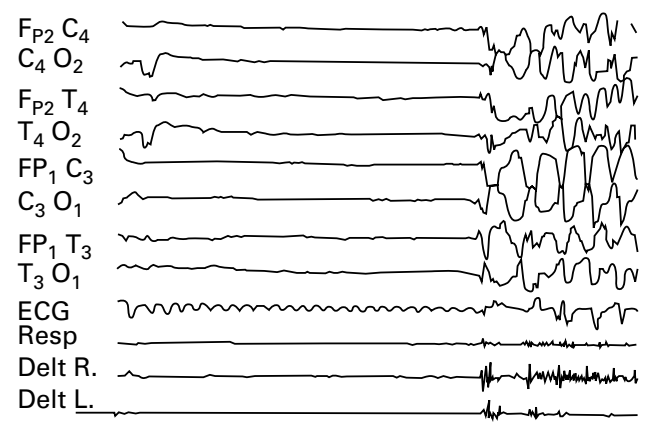

Figure 3 Suppression periods in one patient receiving high doses of clobazam.

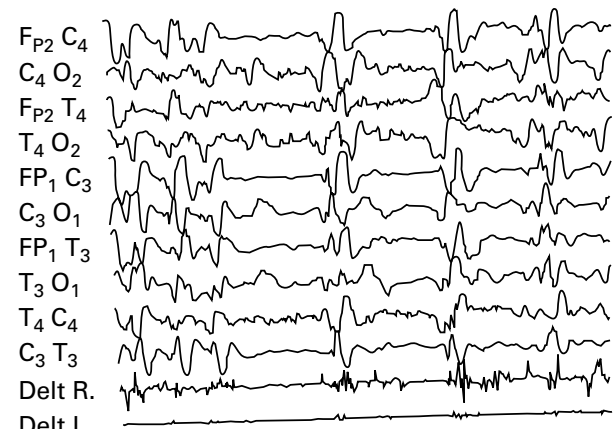

Delt L.

Figure 4 Spasm pattern of seizure.

three patients, mostly in the central areas but also in the temporal areas, and diffuse polyspikes in one patients.

Seizures of various types were recorded in all cases: spasms (fig 4), myoclonia (fig 5), and partial seizures (fig 6) were present on the same tracing, and often intermingled.

Continuous, bilateral, high voltage rhythmic delta slow wave activity, sometimes synchronous with myoclonic jerks, was noticed in four patients (fig 7).

OTHER FINDINGS

Routine metabolic work up (serum electrolytes, glucose, calcium, magnesium, phosphorus, $\mathrm{C}$ reactive protein, arterial gases and blood 


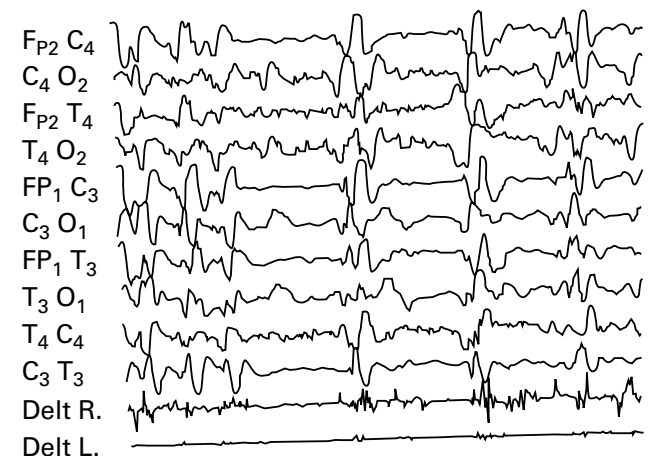

Figure 5 Myoclonic pattern of seizure.

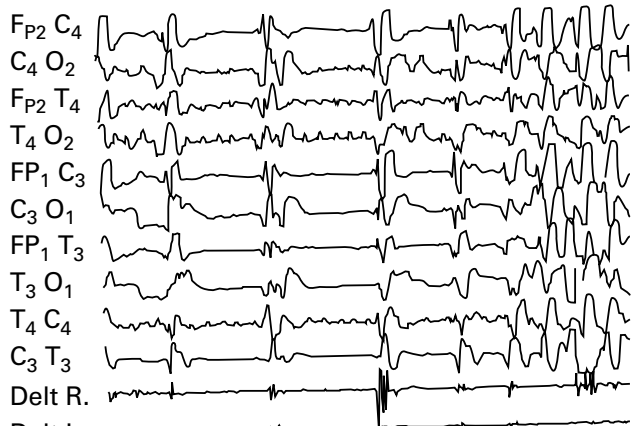

Delt L.

Figure 6 Partial seizure type pattern.

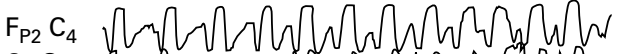
$\mathrm{C}_{4} \mathrm{O}_{2}$ minnm

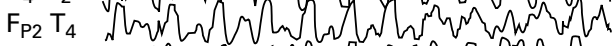
$\mathrm{T}_{4} \mathrm{O}_{2}$, $\mathrm{FP}_{3} \mathrm{C}_{3}$, In



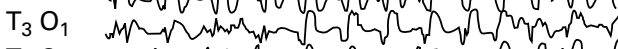

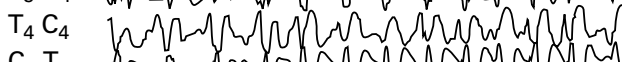
$\mathrm{C}_{3} \mathrm{~T}_{3}$, Delt L.

Figure 7 Continuous bilateral high voltage rhythmic delta slow wave activity in four patients.

count), amino acid and organic acid chromatography were normal. Urine, blood, and cerebrospinal fluid cultures and serology for the main fetal disorders were negative.

Brain computed tomograms were normal in the first week of life, except in one patient who had mild triventricular dilatation.

DIAGNOSIS AND TREATMENT

The age at diagnosis ranged from 3 to 58 days (mean 24 days). The four patients who were the first affected in each given family had received conventional anti-epileptic drugs before pyridoxine dependency was suspected, and seizures had been transiently controlled, for a period of several days, up to 2 weeks.

In all cases pyridoxine resolved clinical symptoms and normalised EEG recordings within a few hours. Four neonates were given pyridoxine intravenously at the dose of $100 \mathrm{mg}$, and immediately after the injection, developed major hypotonia with bradycardia, bradypnoea, and hypothermia, and one patient had to be referred to neonatal intensive care for prolonged apnoea. These unwarranted side effects resolved spontaneously in 4 to 24 hours. The patient who received pyridoxine orally (200 mg/day) had no side effects.

The oral daily maintenance dose of pyridoxine was $200 \mathrm{mg} /$ day. Other anti-epileptic drugs were easily withdrawn and all patients remained free of seizures except one who also required clonazepam for the first eight months of life.

\section{OUTCOME}

Follow up ranged from 2 to 10 years. Pyridoxine was withdrawn in four patients, to confirm pyridoxine dependency, and seizures recurred in all of them. The treatment interruption was done accidentally in the last patient, in whom seizures also recurred. Relapse occurred within 7 days to 3 weeks after pyridoxine was withdrawn. In one child a second attempt to stop pyridoxine failed at the age of 10 years, after 8 years of no seizures; these recurred within two weeks.

At the end of follow up, all patients remained on pyridoxine and were free of seizures. At this point, psychomotor delay was the major concern for all patients. It was severe in three, moderate in one, and mild in one. Motor sequelae were less severe as all patients were walking by the ages of 3 to 5 years.

Two patients exhibited abnormal density of the white matter and ventricular dilatation. The latter had been noticed at birth in one patient who required ventriculo-peritoneal shunting for aqueduct stenosis at 2 years of age.

\section{Discussion}

Pyridoxine dependent epilepsy is probably underdiagnosed. Diagnosis is usually not suspected before neonatal seizures are considered clearly resistant to conventional antiepileptic drugs. Therefore, pyridoxine treatment is often started after a period of some delay. We found a characteristic clinical and EEG pattern highly suggestive of this disorder, which might help to identify the diagnosis earlier, and thus to avoid inappropriate treatment and delayed diagnosis. A family history of miscarriage, stillbirth, and neonatal death during status epilepticus is indicative. ${ }^{25} 8^{11-13}$ Potential intrauterine seizures are also helpful clues. ${ }^{291214}$ Such movements are described in almost $20 \%$ of reported cases, ${ }^{15}$ and in one patient of our series. Fetal distress was present in three patients with a meconium stained amniotic fluid. Such misleading features have been mentioned ${ }^{713}$ and thus, documented neonatal asphyxia does not rule out pyridoxine dependency.

Seizures occur very early, mostly in the first days of life..$^{2-5}{ }^{14}{ }^{16-20}$ Although no specific type is involved, the mixture of a great variety of different types of seizures from the very first days of life is unusual, and has been a striking feature shared by all patients in this series.

Clinical anomalies other than seizures are characteristic and might be of diagnostic help. Hyperexcitability and unremitting agitation are 
a constant hallmark in all reported cases, and are highly predictive..$^{2812131521}$ Hypotonia, feeding difficulties, and lack of visual alertness have also been reported..$^{13} 1522$

EEG recordings before pyridoxine administration produce a suggestive pattern. Continuous diffuse high voltage rhythmic delta slow waves, with myoclonic jerks are typical. A similar pattern has been reported in several pyridoxine dependent patients, but it occurs only in bursts and intermingled with spikes. ${ }^{23}$ Such continuous delta activity has also been reported in rats with pyridoxine depletion. ${ }^{24}$

Delay before diagnosis and specific treatment is common in all sporadic cases, especially in cases with partial response to conventional anti-epileptic drugs. ${ }^{725}$ In the 76 patients, including sporadic and familial cases, reviewed by Haeggenli, the mean age at the time of diagnosis was 3.5 months, ranging from 1 day to 22 months. ${ }^{15}$ As in our series, the diagnosis of familial cases was made earlier.

Various types of seizures occur in neonates. Epileptic myoclonus and partial seizures have been reported in other series, ${ }^{2891923}$ more often than spasms. ${ }^{78}$ Four patients in this series had clear spasms, and this pattern may be quite common, although rarely reported because of lack of consensus in the terminology of neonatal seizures.

When pyridoxine is administered intravenously, there are adverse side effects, consisting of immediate bradycardia, bradypnoea, lethargy and hypothermia, which often develop in cases of pyridoxine dependency. These manifestations are usually mild and reversible within a few hours ${ }^{12} 1522{ }^{23}$ but rarely require artificial ventilation $^{14}$ and one patient in our series required transfer to intensive care. These symptoms may follow intramuscular administration $^{7}$ and even, very rarely, oral administration. ${ }^{12}$ They are thought to result from sudden and massive release of GABA. ${ }^{12}$ However, the two patients in our series who received pyridoxine orally at the initial dose of $200 \mathrm{mg}$, did not have such adverse effects, and the therapeutic benefit was similar to that following intravenous administration. Therefore, a pyridoxine trial should be performed orally with $200 \mathrm{mg}$ as the initial dose, and the intravenous route restricted to patients with status epilepticus. The response occurs no more than 12 hours after oral administration, and therefore the administration of $200 \mathrm{mg} /$ day for two consecutive days is enough to preclude or confirm the diagnosis.

Most children with pyridoxine dependent epilepsy are mentally retarded. ${ }^{12}{ }^{15-23}$ Rarely do patients diagnosed and treated late develop normally, but despite early treatment most others sustain psychomotor retardation. ${ }^{2}{ }^{15}$ Early treatment may, however, decrease the severity or frequency of subsequent encephalopathy. Although pyridoxine supplementation to the mother during pregnancy was reported not to have prevented retardation in two cases, ${ }^{226}$ in one other case the child was not retarded. ${ }^{3}$ This is evident in one familial case of our series. The youngest brother of cases 4 and 5 (table 1) did not have neonatal seizures. His mother received pyridoxine supplementation during pregnancy, as did he, from birth. His first seizure occurred 6 months after pyridoxine had been withdrawn in order to confirm the diagnosis of pyridoxine dependency. The psychomotor development of these three brothers was correlated with the age at diagnosis as the eldest who was treated at 1 month of age, has severe psychomotor retardation, the middle brother, treated at 4 days of life, had only mild retardation, and the youngest, treated in utero and from birth, was normally developed at 1 year of age. Thus we believe that brain damage may occur before birth as may seizures. Therefore, treatment of the pregnant mother should be advised.

Oral maintenance treatment is recommended, but a relatively wide dose range is advised: from 2 to $300 \mathrm{mg} /$ day. $^{72}{ }^{1528}$ A daily dose of $200 \mathrm{mg}$ was sufficient in our series, and an increase of pyridoxine was not needed during intercurrent illness, although this has been suggested. ${ }^{781322}$ No toxicity or side effects were noticed with this dose..$^{15}$ When withdrawal of pyridoxine is used as a diagnostic test or done accidentally, seizures recur with concomitant EEG deterioration. Thus permanent pyridoxine dependency involves all reported cases. $^{781322}$

Several cases of delayed onset after the neonatal period have been reported, ${ }^{4-9} 29$ and some authors suggest considering this diagnosis in all children under 18 months of age with intractable seizures of unknown aetiology. ${ }^{7811}$ The clinical spectrum, including typical and atypical presentations, suggests a heterogenous disorder.

Pyridoxine dependent seizures are a rare autosomal recessive disorder. Many familial cases are reported with consanguineous parents. $^{781112}$ It is probably due to altered binding of PLP to cerebral GAD, leading to a defect in brain GABA production..$^{30}$ Indeed, the GABA concentration is decreased in the brain $^{13}$ and cerebrospinal fluid of affected patients, ${ }^{21}{ }^{31}$ and increases in the cerebrospinal fluid after pyridoxine administration..$^{32}$ In one recent study, PLP dependent GAD activity was reduced in the fibroblasts in the renal biopsy specimen of one affected patient. ${ }^{33}$ Therefore, the evidence that brain GAD activity is deficient in this disease is still circumspect. ${ }^{14}$

Molecular genetics should soon identify the gene concerned and contribute to early diagnosis, treatment, and prenatal diagnosis. A sequence homologous to glutamic acid decarboxylase cDNA is present on mouse chromosomes 2 and $10 .^{34}{ }^{35}$

1 Hunt AD, Stokes J, Stround HH. Pyridoxine dependency: report of a case of intractable convulsions in an infant controlled by Pyridoxine. Pediatrics 1954;13:1405.

2 Bejsovec M, Kulenda Z, Ponca E. Familial intrauterine convulsions in Pyridoxine dependency. Arch Dis Child 1967;42:201-7.

3 Scriver CR. Vitamin B6 dependency and infantile convulsions. Pediatrics 1960;26:62-74.

4 Scriver CR, Gibson KM. Disorders of beta and gamma amino-acids in free and peptide linked forms. In: The metabolic and molecular bases of inherited disease. 7 th edn. New York: McGraw Hill, 1995:1349-60.

5 Waldinger C, Berg RB. Signs of Pyridoxine dependency manifest at birth in siblings. Pediatrics 1963;32:161-8.

6 Bachman DS. Late onset Pyridoxine dependency convulsions. Ann Neurol 1983;14:692-3.
sitionman 
7 Bankier A, Turner M, Hopkins IJ.Pyridoxine dependent seizures- a wider clinical spectrum. Arch Dis Child 1983 58:415-18

8 Goutières F, Aicardi J. Atypical presentation of Pyridoxine dependent seizures: A treatable cause of intractable epilepsy in infants. Ann Neurol 1984;17:117-20.

9 Krishnamoorthy KS. Pyridoxine dependency seizure: report of a rare presentation. Ann Neurol 1983;13:103-4.

10 Baumeister FAM, Gsell W, Shin YS, Egger J.Glutamate in Pyridoxine epilepsy: neurotoxic glutamate concentration in the cerebrospinal fluid and its normalization by Pyridoxine. Pediatrics 1994;3:318-21.

11 Jardim LB, Pires RF, Martins CES, Vargas CR, Vizioli J, Kliemann FAD, Giugliani R. Pyridoxine dependent seizures associated with white matter abnormalities. Neuropediatrics 1994;25:259-61.

12 Kroll JS. Pyridoxine for neonatal seizures: an unexpected danger. Dev Med Child Neurol 1985;27:369-82.

13 Lott IT, Coulombe T, Di Paolo RV, Richardson EP, Levy HL. Vitamin B6-dependent seizures: pathology and chemical findings in brain. Neurology 1978;28:47-54.

14 Heeley A, Pugh RJP, Clayton BE, Sheperd J, Wilson J. Pyridoxal metabolism in vitamine B6-responsive convulsions in early infancy. Arch Dis Child 1978;53:794-802.

15 Haeggenli CA, Girardin E, Paunier L. Pyridoxine dependent seizures: clinical and therapeutic aspect. Eur $f$ Pediatrics 1991;150:452-5.

16 Bessey OA, Adam DJD, Hansen AE.Intake of vitamin B6 and infantile convulsions: a first approximation of requierments in children. Pediatrics 1957;20:33-44.

17 Clarke TA, Saunders BS, Feldman B. Pyridoxinedependent seizures requiring high doses of Pyridoxine fo control. Am 7 Dis Child 1979;133:963-5.

18 Dolina S, Peeling J, Sutherland G, Pillay N, Greenberg A.Effect of sustained Pyridoxine treatment on seizure susceptibility and regional brain amino acid levels in genetically epilepsy prone BALB/c mice. Epilepsia 1993; 34:33-42.

19 French JH, Grueter BB, Druckman R, O'Brien D. Pyridoxine and infantile myoclonic seizures. Neurology ine and infan

20 Gentz J, Hamfelt A, Johansson S, Lindstedt S, Persson B, Zetterström R. Vitamin B6 metabolism in pyridoxine dependency with seizures. Acta Paediatrica Scandinavica $1967 ; 56: 17-26$

21 Gospe SM, Olin KL, Keen CL. Reduced GABA synthesis in Pyridoxine-dependent seizures. Lancet 1994; 343:1133-4
22 Tanaka R, Okumura M, Arima J, Yamakura S, Momoi T. Pyridoxine-dependent seizures: report of a case with atypical clinical features and abnormal MRI scans. $\mathcal{F}$ Child Neurol 1992;7:24-8.

23 Mikati MA, Trevathan E, Krishnamoorthy KS, Lombroso C. Pyridoxine dependent epilepsy: EEG investigations and long term follow-up. Electroencephalography and Clinical Neurophysiology 1991;78:215-21.

24 Sharma SK, Dakshinamurti K. Seizure activity in Pyridoxine deficient adult rats. Epilepsia 1992;33:235-47.

25 Swaiman KF, Milstein JM.Pyridoxine dependency and Penicillamine. Neurology 1970;20:78-81.

26 Iiuma K, Narisawa K, Yamauchi N, Yoshida T, Mizuno T. Pyridoxine dependent convulsion: effect of Pyridoxine therapy on E.E.G. Tohuku fournal of Experimental Medecine 1971;105:19-26.

27 Pietz J, Benninger C, Schäfer H, Sontheimer D, Mittermaier G, Rating D. Treatment of infantile spasms with high-dosage vitamin B6. Epilepsia 1993;34:757-63.

28 Petit RE. Pyridoxine dependency seizures: report of a case with unusual features. F Child Neurol 1987;2:38-40.

29 Emery ES. An unusual case of Pyridoxine resonsive seizures (abstract). Ann Neurol 1984;16:395.

30 Scriver CR, Whelan DT. Glutamic acid decarboxylase (GAD) in mammalian tissue outside the nervous system, and its possible relevance to hereditary vitamin B6 dependency with seizures. Ann NY Acad Sci 1969.

31 Kurlemann G, Ziegler R, Gruneberg M, et al. Disturbance of GABA metabolism in Pyridoxine dependent seizures. Neuropediatrics 1992;23:257-9.

32 Kurleman G, Loscher W, Dominick HC, Dominick GD. Disappearance of neonatal seizures and low CSF GABA levels after treatment with vitamin B6. (1987). Epilepsy Research 1978;1:152-9.

33 Yoshida T, Tada K, Arakawa T. Vitamin B6 dependency of glutamic acid decarboxylase in the kidney from a patient with vitamin B6 dependent convulsion. Tohoku fournal of Experimental Medicine 1971:195-202.

34 Brillant MH, Szaba G, Katarova Z, et al. Sequences homologous to glutamic acid decarboxylase cDNA are present on mouth chromosome 2 and 10. Genomics 1990; 6:115-21.

35 Erlander MG, Tillakaratne JK, Feldblum S, Patel N, Tobin AJ. Two genes encode distinct glutamate decarboxylases. Neuron 1991;7:91-8. 